\title{
Sensory involvement in the SOD1-G93A mouse model of amyotrophic lateral sclerosis
}

\author{
Yan-Su Guo ${ }^{1,2}$, Dong-Xia Wu, ${ }^{1,2}$, \\ Hong-Ran $\mathrm{Wu}^{1,2}$, Shu-Yu Wu, ${ }^{1,2}$, \\ Cheng Yang ${ }^{1,2}$, Bin $\mathrm{Li}^{1}$, Hui Bu ${ }^{1,2}$, \\ Yue-sheng Zhang ${ }^{3}$ and Chun-Yan $\mathrm{Li}^{1,2,4}$ \\ ${ }^{1}$ Department of Neurology \\ ${ }^{2}$ Hebei Province Key Laboratory of Neurology \\ Institute of Cardiocerebrovascular Disease \\ The Second Hospital of Hebei Medical University \\ Shijiazhuang, Hebei 050000, China \\ ${ }^{3}$ Department of Chemoprevention \\ Roswell Park Cancer Institute \\ Elm and Carlton Streets \\ Buffalo, NY 14263, USA \\ ${ }^{4}$ Corresponding author: Tel, 86-311-87064024; \\ Fax, 86-311-87064024; E-mail, chunyanli5@yahoo.com.cn \\ DOI 10.3858/emm.2009.41.3.017
}

Accepted 6 November 2008

Abbreviations: ALS, amyotrophic lateral sclerosis; DF, dorsal funiculus; DR, dorsal root; DRG, dorsal root ganglia; SOD1, superoxide dismutase 1; VR, ventral root

\begin{abstract}
A subset of patients of amyotrophic lateral sclerosis (ALS) present with mutation of $\mathrm{Cu} / \mathrm{Zn}$ superoxide dismutase 1 (SOD1), and such mutants caused an ALSlike disorder when expressed in rodents. These findings implicated SOD1 in ALS pathogenesis and made the transgenic animals a widely used ALS model. However, previous studies of these animals have focused largely on motor neuron damage. We report herein that the spinal cords of mice expressing a human SOD1 mutant (hSOD1-G93A), besides showing typical destruction of motor neurons and axons, exhibit significant damage in the sensory system, including Wallerian-like degeneration in axons of dorsal root and dorsal funiculus, and mitochondrial damage in dorsal root ganglia neurons. Thus, hSOD1-G93A mutation causes both motor and sensory neuropathies, and as such the disease developed in the transgenic mice very closely resembles human ALS.
\end{abstract}

Keywords: amyotrophic lateral sclerosis; mutation; nerve degeneration; spinal cord; spinal nerve roots; superoxide dismutase 1

\section{Introduction}

Amyotrophic lateral sclerosis (ALS), also known as Lou Gehrig's disease, is a progressive and fatal neurodegenerative disease. The hallmark of ALS is selective loss of motor neurons in the spinal cord, brainstem and cerebral cortex, resulting in muscle weakness and atrophy throughout the body. However, sensory neuropathy, including axonal loss in sensory nerve biopsy, abnormalities in somatosensory evoked potentials, reduced spinal cord conduction velocity, diminished vibration sense and/or abnormal touch-pressure sensation were also detected in up to 23\% ALS patients (Dyck et al., 1975; Georgesco et al., 1994; Matsumoto et al., 1999; Isaacs et al., 2007; Pugdahl et al., 2007), implying that ALS is a multi-system neurodegenerative disease. Family history is indicated in 5 $10 \%$ cases, but the sporadic and familial cases are clinically indistinguishable (Andersen, 2006), suggesting similar underlying mechanisms of neurodegeneration. Of the familial cases, about $20 \%$ present mutations in the gene that codes the cytosolic $\mathrm{Cu} / \mathrm{Zn}$ superoxide dismutase 1 (hSOD1) (Rosen et al., 1993). Many of the hSOD1 mutants retain the enzymatic activity, but expression of such a mutant caused apoptosis in cultured neural cells and ALS-like disorder in animals while expression of the wild-type hSOD1 was not harmful (Gurney et al., 1994; Rabizadeh et al., 1995; Tu et al., 1996), although one study reported that expressing high levels of wild-type hSOD1 in mice was also mildly toxic to motor neurons (Jaarsma et al., 2000). These and other similar studies have led to the widely held belief that SOD1 mutation plays a major role in the pathogenesis of at least some familial ALS in humans. The mechanism by which mutant hSOD1 causes motor neuron death remains poorly understood. Interestingly, sensory impairment was also detected in a patient of familial ALS with a missense mutation (Gly93 $\rightarrow$ Ser) in exon 4 of hSOD1 (Kawata et al., 1997).

Because expression of a hSOD1 mutant in animals develops an adult-onset and progressive loss of motor function and motor neurons, these transgenic animals have become a widely used ALS model. However, previous studies of these animals 
have focused largely on the motor system. Although degeneration of spinal cord DR axons in these animals has been mentioned in several previous reports (Sun et al., 2002; Fischer et al., 2005), detailed study about the damages in the sensory neurons and axons has not been reported. In view of the presence of sensory neuropathy in a large number of ALS patients, information on the sensory system of these mice is scarce but important. In the present study we have investigated the potential alterations of spinal cord sensory neurons and axons in transgenic mice expressing a hSOD1 mutant.

\section{Results}

The present study was performed in transgenic mice expressing a hSOD1 mutant where two adjacent nucleotides of exon 4/codon 93 of SOD1 were mutated resulting in replacement of glycine by alanine (hSOD1-G93A), which occurs in some familial ALS patients (Rosen et al., 1993). Non-transgenic littermates were used as a control. The histopathological examinations were focused on the lumbar region of the spinal cord (see Figure 1 for specific areas examined), since loss of motor neurons in ALS is most prominent in this region.

\section{Progressive motor deficit and loss of motor neurons in hSOD1-G93A mice}

The hSOD1-G93A mice began to show tremor in one or both hind limbs at $90 \sim 100$ days of age and then presented progressive loss of motor functions, as indicated by gait abnormality and failure to extend a limb while being held in the air by their tails. These abnormalities were accompanied by muscle atrophy and loss of muscle strength. The animals became moribund at $131.3 \pm 9.4$ and $137.8 \pm 11.9$ days of age for male and female mice respectively (mean $\pm \mathrm{SD}, n=18-19$ ): severe

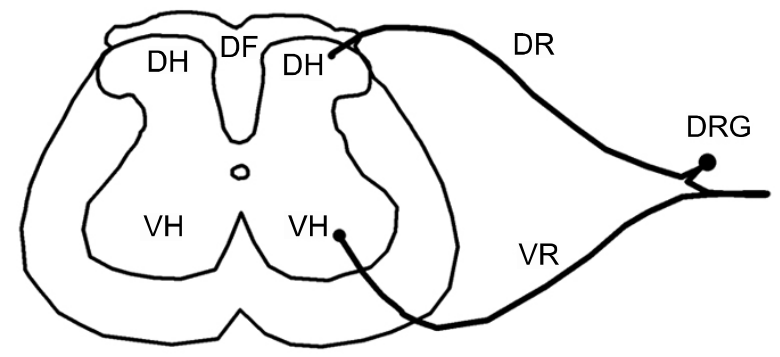

Figure 1. Areas of spinal cord, including dorsal funiculus (DF), dorsal horn (DH), dorsal root (DR), dorsal root ganglia (DRG), ventral horn (VH) and ventral root (VR). paralysis (failure to right itself within 30 seconds when placed on its side) and loss of up to $40 \%$ of their body weight. The age difference in reaching the moribund state between the male and female mice was not statistically significant. The animals were euthanized when reaching the moribund state, which is referred to as end stage throughout this report. Moreover, the number of ventral horn motor neurons in the spinal cords of hSOD1-G93A mice at end stage decreased $61.8 \%$, compared with that in the littermates (Table 1). In contrast, no abnormalities were observed in the non-transgenic littermates during the same experimental period. These results confirm the presence of motor neuron degeneration in the hSOD1-G93A mice and are consistent with previous findings (Gurney et al., 1994; Feeney et al., 2001; Zang and Cheema, 2002). The number of dorsal horn sensory neurons in SOD1-G93A mice was not significantly different from that in their non-transgenic littermates (Table 1), consistent with the prevailing view that expression of hSOD1-G93A causes motor neuron disease.

\section{Damage of both ventral and dorsal roots in hSOD1-G93A mouse spinal cord}

The ventral root (VR) derives from ventral horn motor neurons, whereas the dorsal root (DR) derives from sensory neurons in DRG (Figure 1). A previous study showed that both fast and slow axonal transports were impaired in the VR of hSOD1-G93A mice (Zhang et al., 1997). We observed extensive and severe degeneration of $V R$ in hSOD1-G93A mice at end stage, but not in the non-transgenic littermates at similar age (Figure 2). Similar changes also occurred in the DR of hSOD1G93A mice, although perhaps somewhat less

Table 1. Number of spinal cord neurons in mice with and without expression of hSOD1-G93A.

\begin{tabular}{lcc}
\hline \multirow{2}{*}{\multicolumn{1}{c}{ Mice }} & \multicolumn{2}{c}{ Number of neurons per tissue section } \\
\cline { 2 - 3 } & Motor neuron & Sensory neuron \\
\hline Littermate & $12.3 \pm 1.8$ & $70.7 \pm 8.0$ \\
hSOD1-G93A & $4.7 \pm 2.0^{*}$ & $68.3 \pm 8.5$ \\
\hline
\end{tabular}

Lumbar enlargements were harvested from hSOD1-G93A mice at end stage and their non-transgenic littermates at the same age. There were 3 animals in each group and 12-15 sections were counted from each animal. Transversal sections $(20 \mu \mathrm{m})$ were cut on a Leica VT $1000 \mathrm{~S}$ vibratome. Motor neurons and sensory neurons were identified by immunohistochemical staining with SMI-32 and calretinin respectively. SMI-32-stained neurons in the ventral horn with a diameter of $\geq 25 \mu \mathrm{m}$ were counted as motor neurons. Calretinin-positive neurons in the dorsal horn were counted as sensory neurons. Each value is a mean $\pm S D$. ${ }^{*} P$ $<0.05$ vs Littermate. 


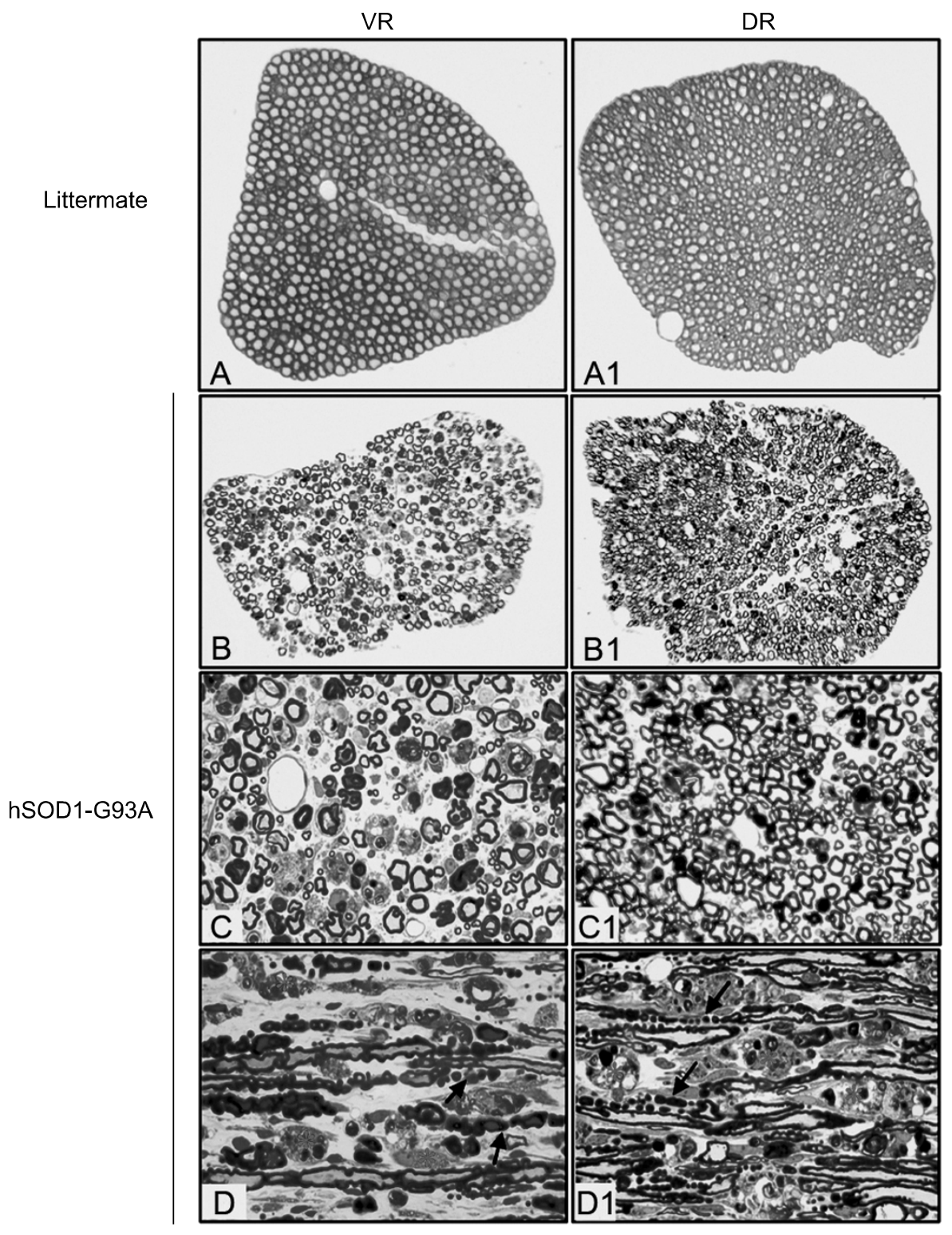

Figure 2. Semithin sections showing degeneration of VR and DR in hSOD1G93A mice. Tissues stained with Toluidine blue were obtained from hSOD1G93A mice at end stage and their nontransgenic littermates at the same age. $A-D$ was from VR and A1-D1 from DR. Many degenerated nerve fibers were observed in both VR and DR, and density of myelinated nerve fibers were reduced, especially in the VR. Arrows point to bead-like ovoid formed by degradated myelin sheath, and asterisks indicate macrophage-like cells ( $D$ and $D 1$ ). The data are representative of all animals examined, at least 3 animals in each group. $A, B, A 1$, and $B 1: 200 \times ; C, D, C 1$, and D1: $400 \times$. profound (Figure 2). In the semithin sections (1 $\mu \mathrm{m})$ stained by toluidine blue, different stages of axonal disintegration could be observed in the transverse sections of both VR (Figure $2 \mathrm{~B}$ and $\mathrm{C}$ ) and DR (Figure 2B1 and C1). The density of myelinated nerve fibers was greatly reduced (Table 2 ). In the longitudinal sections, similar as seen in the transverse sections, the number of myelinated nerve fibers was greatly decreased. Wallerian-like degeneration was universal. Some myelin sheath formed bead-like ovoids (indicated by arrows in Figure 2D and D1). Moreover, degraded myelin debris appeared to be sequestered in phagocytes (indicated by the asterisks in Figure 2D and D1), suggesting that these cells might be assuming the debris-clearing task.
Table 2. Number of ventral and dorsal root axons.

\begin{tabular}{lccc}
\hline \multicolumn{1}{c}{ Age groups } & $\mathrm{n}$ & $\begin{array}{c}\text { Number of } \\
\text { total axons }\end{array}$ & $\begin{array}{c}\text { Number of } \\
\text { degenerated axons }\end{array}$ \\
\hline Littermate VR & 3 & $323.00 \pm 62.19$ & $0.67 \pm 0.58$ \\
60d G93A VR & 3 & $291.33 \pm 23.50$ & $23.33 \pm 1.53^{*}$ \\
120d G93A VR & 3 & $165.67 \pm 5.69^{*}$ & $42.67 \pm 2.08^{*}$ \\
End G93A VR & 3 & $173.33 \pm 4.73^{*}$ & $65.00 \pm 7.00^{*}$ \\
Littermate DR & 3 & $444.67 \pm 64.93$ & $1.33 \pm 0.58$ \\
60d G93A DR & 3 & $382.67 \pm 40.67$ & $17.33 \pm 2.52^{*}$ \\
120d G93A DR & 3 & $378.33 \pm 12.06$ & $28.33 \pm 4.16^{*}$ \\
End G93A DR & 3 & $197.33 \pm 2.08^{*}$ & $60.67 \pm 1.53^{*}$ \\
\hline
\end{tabular}

Number of total and degenerated myelinated axons in the lumbar ventral and dorsal root were counted at $400 \times$ visual field on toluidine blue stained semi-thin sections ( $n=3$ fields from 3 mice). Number of total and degenerated VR and DR myelinated axons in G93A at $60 \mathrm{~d}, 120 \mathrm{~d}$ and end stage (End) were compared with their littermates respectively. Each value is a mean \pm SD. ${ }^{*}, P<0.05$. 


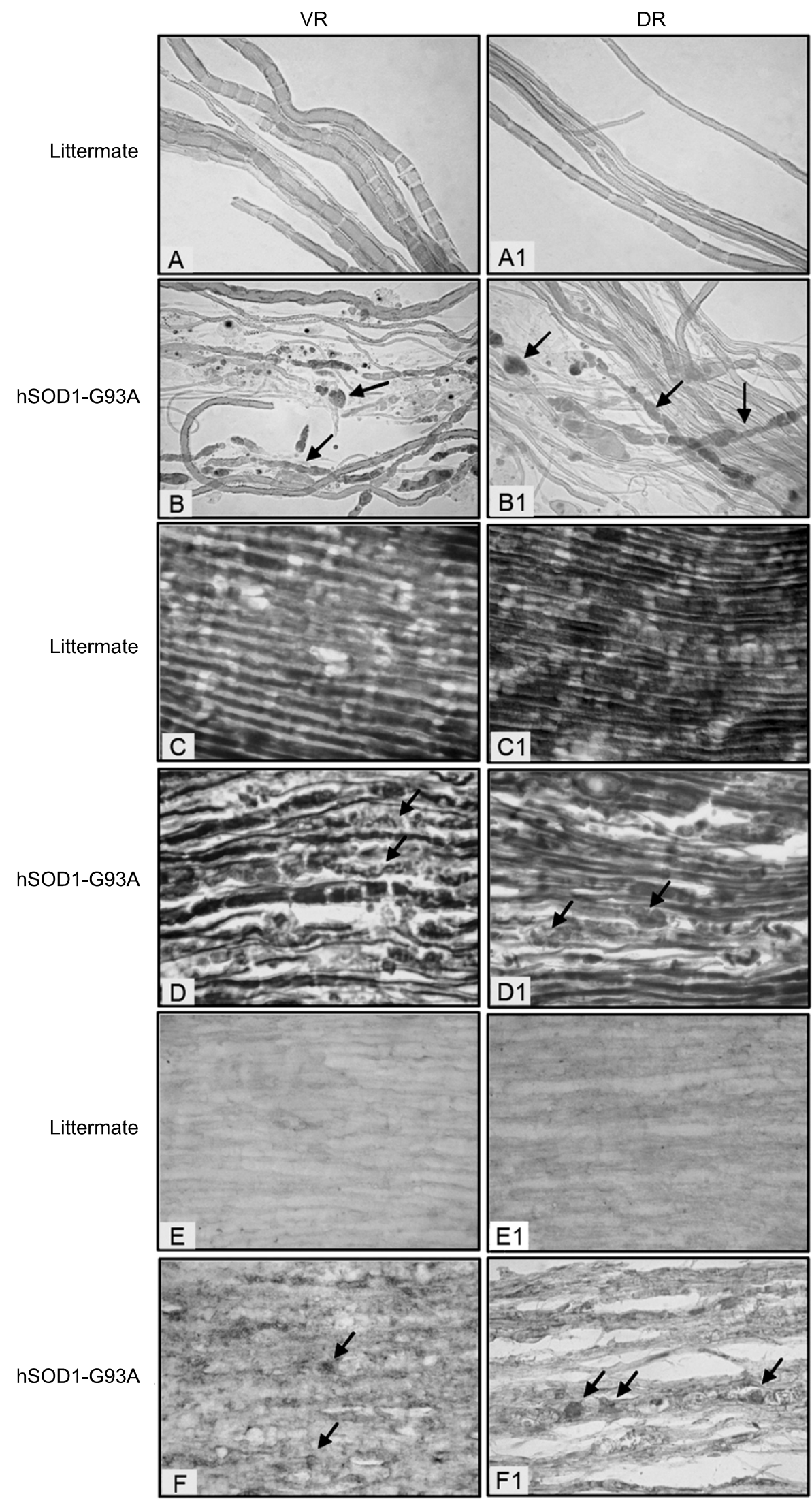

Figure 3. A, A1, B and B1: teased fibers, showing Wallarian-like degeneration in VR and DR of hSOD1-G93A mice at end stage. Arrows indicate Wallerian-like degeneration of myelinated nerve fibers forming ovoids. $400 \times$. C, C1, D and D1: Naumenko-Feigin silver stain. Arrows point to degenerating axons in VR and DR of hSOD1-G93A mice at end stage. $400 \times$. E, E1, F and F1: immunohistochemical staining of CD68. Arrows indicate $\mathrm{CD} 68$ positive cells between the nerve fibers of VR and DR of hSOD1G93A mice at end stage. $400 \times$. The data are representative of all animals examined, at least 3 animals in each group. 
Using the teasing fiber method, nerve fibers in the VR and DR of non-transgenic littermates were shown to possess intact myelin sheath (lateral and dense color) and axon (central and light color) with uniform diameter within each fiber and normal internodes (Figure $3 \mathrm{~A}$ and $\mathrm{A} 1$ ). In contrast, Wallerianlike degeneration (discontinuous and/or swollen axons, and fragmented myelin sheath) was seen in both VR and DR of hSOD1-G93A mice (Figure 3B and B1). Different stages of degeneration of myelinated fibers were present, with some fibers forming bead-like structures and others collapsing into myelin ovoid or debris. Displayed by the Naumenko-Feigin silver staining, the axons in the VR and DR of non-transgenic littermates were continuous and uniform in caliber (Figure $3 C$ and $C 1$ ), whereas the axons in these regions of hSOD1G93A mice were swollen and disintegrated (Figure

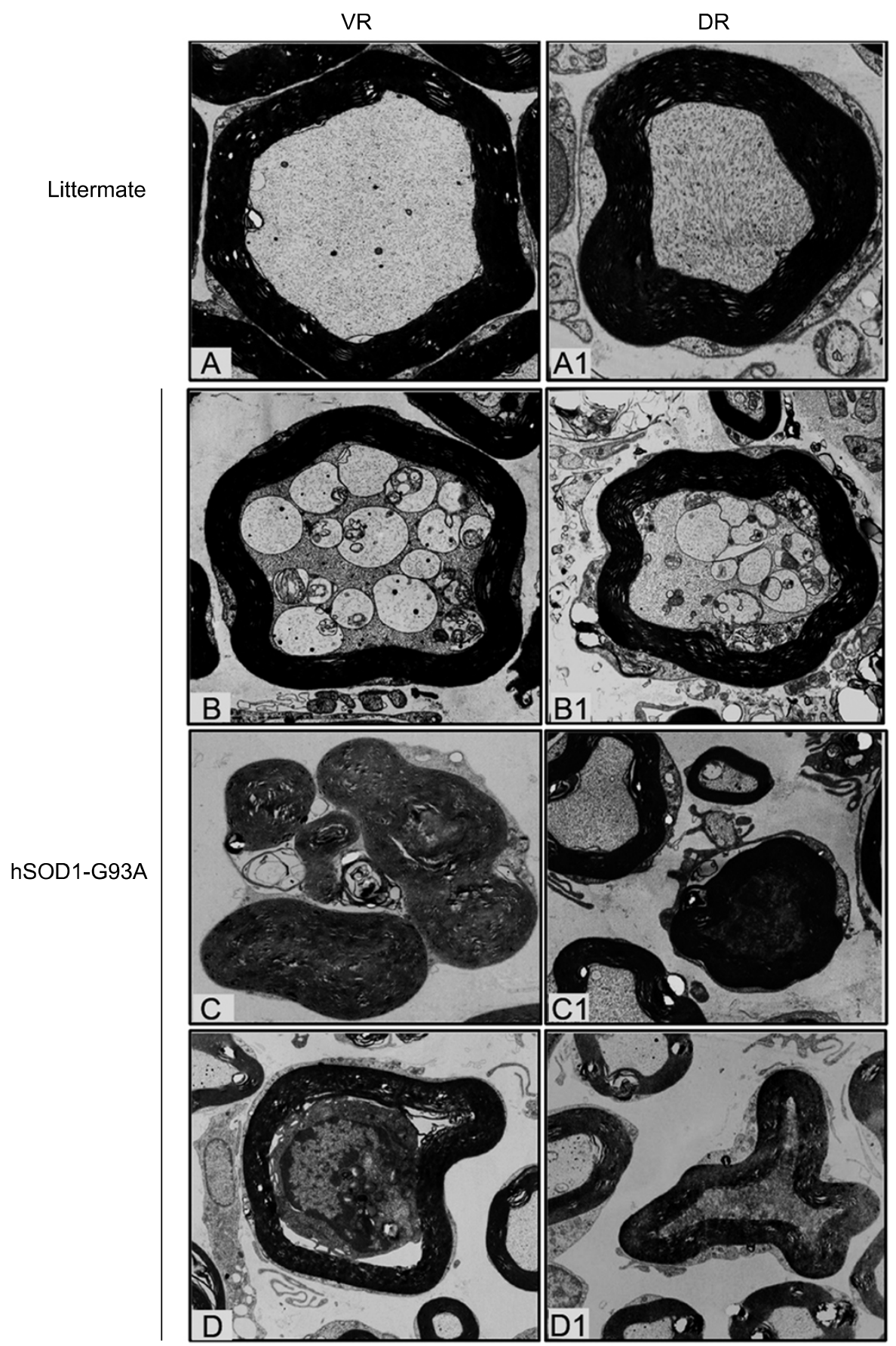

Figure 4. Transmission electron microscopic examination on VR and DR of hSOD1-G93A mice and their littermates. $4000 \times$. A and A1: normal axon and myelin sheath. B and B1: showing numerous vacuoles and swollen mitochondria in the axoplasm; C, C1 and D1: indicating collapse and degradation of myelin sheath with condensed or absent axon in the central part; $D$ shows a macrophage-like cell within the myelin sheath occupying the axon. The data are representative of all animals examined, at least 3 animals in each group. 
3D and D1). Furthermore, because some of the above results suggested that macrophages might be involved in the axonal damage (Figure 2D and D1), we performed immunohistochemical staining of CD68, which is a glycoprotein and is specifically expressed on macrophages. There were many CD68-positive spots (cells) in the VR and DR of hSOD1-G93A mice (Figure 3F and F1), whereas it was negative (only background staining) in the tissues of non-transgenic littermates (Figure $3 \mathrm{E}$ and E1). This finding strongly suggests that macrophages are indeed involved in the axonal degeneration.

While the transmission electron micrographs of VR and DR of non-transgenic littermates showed a relatively homogenous axoplasm and intacted myelin sheath (Figure $4 A$ and $A 1$ ), the axoplasm of VR and DR of hSOD1-G93A was occupied by vacuoles and swollen mitochondria (Figure 4B and B1) and accompanied by degenerated and disappeared axons, myelin sheath collapsed and de- gradated into ovoids (Figure 4C, C1 and D1). In a few of cases, the axons were occupied completely or partly by a cell resembling a macrophage (Figure 4D). It was notable that these types of changes occurred in both VR and DR.

\section{Damage of DRG neurons}

Because the axons in DR were significantly damaged in hSOD1-G93A mice, we next examined the neurons in DRG. There did not appear to be a clear decrease in the number of DRG neurons in hSOD1-G93A mice (Figure 5A and $B$ ), based on the comparison of multiple sections of DRG from both hSOD1-G93A mice and their non-transgenic littermates. However, numerous vacuoles existed in many DRG neurons of hSOD1-G93A mice but not in those of the control animals (Figure $5 \mathrm{C}$ and $\mathrm{D}$ ). Moreover, numerous DRG neurons of hSOD1-G93A mice showed swollen mitochondria under transmission electron microscope (Figure 5E and F), further

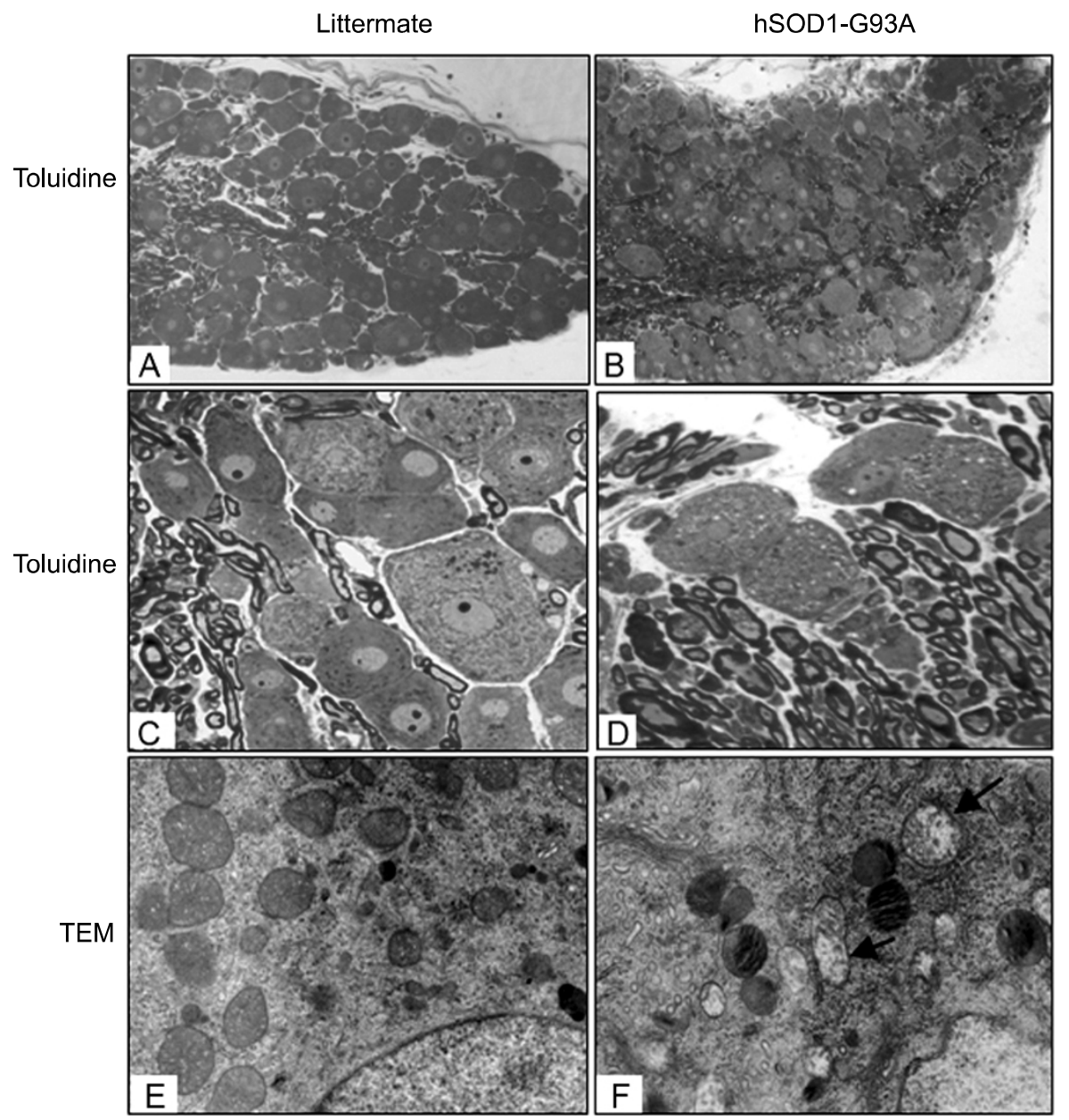

Figure 5. DRG degeneration in hSOD1G93A mice. Tissues were obtained from hSOD1-G93A mice at end stage and their non-transgenic littermates at the same age. A-D: Toluidine blue staining. $A$ and $B: 100 \times ; C$ and D: $400 \times$. E and $\mathrm{F}$ : transmission electron microscopy. Arrows indicate swollen mitochondria in the DRG neurons of hSOD1-G93A mice. $15,000 \times$ 
documenting degeneration of these neurons.

\section{Degeneration of dorsal funiculus (DF)}

In addition to the damage of DR and DRG, hSOD1-
G93A mice also exhibited severe DF degeneration. Staining the tissue sections with monoclonal antibody SMI-31, which recognizes phosphorylated neurofilaments, revealed extensive and severe loss of phosphorylated neurofilaments in DF, mainly in
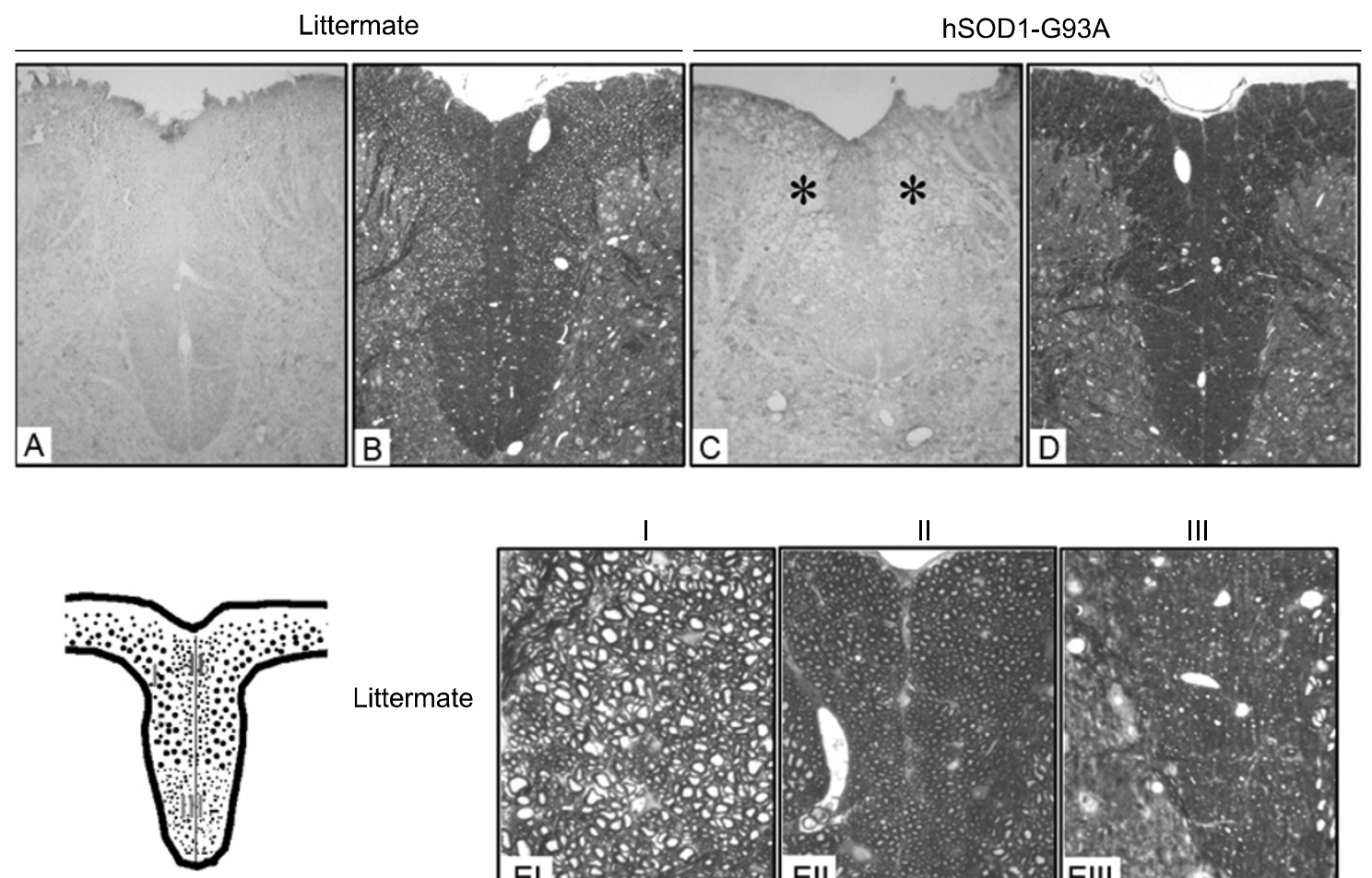

Littermate
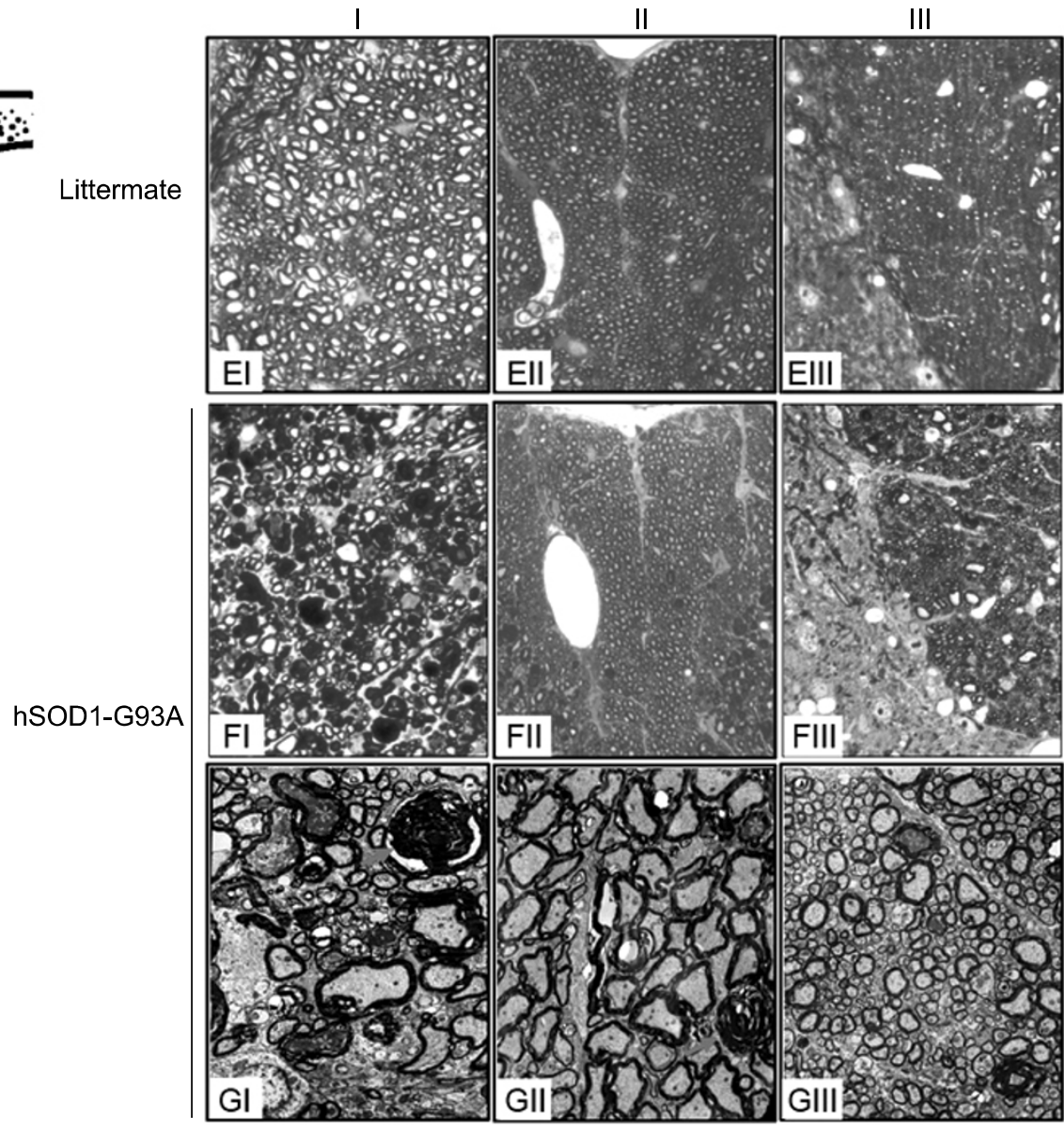

Figure 6. DF degeneration in hSOD1-G93A mice. Tissues were obtained from hSOD1-G93A mice at end stage and their non-transgenic littermates at the same age. A-D: $100 \times ; E$ and F: $400 \times$. G: $4000 \times$. A and C: immunohistochemical staining with SMI-31. Asterisks show spongy degeneration of DF in hSOD1-G93A mice. B, D, E and F: toluidine blue staining. G: transmission electron microscopy. Arrows show disaggregated myelin sheath forming ovoids with the central axons disappeared. Asterisks indicate electron-densed axons in DF of hSOD1-G93A mice. The data are representative of all animals examined, at least 3 animals in each group. 
Region I (Figure 6A and C). Staining the tissue sections with toluidine blue showed the presence of numerous dense deposits in Region I of hSOD1G93A mice (Figure 6B and D), which under high magnification were seen as decomposed nerve fibers with the normal ring-like myelin sheath and the central axon (Figure 6El-III) being replaced by dense spheroids (Figure 6FI). Under transmission electron microscope, the dense spheroids showed by toluidine staining were verified as disintegrated myelin sheaths with the central axon disappeared. There were still many axons represented as high-electron density with their myelin sheaths collapsed more or less (indicated as asterisks in Figure 6GI). Region II and III were mostly uninvolved with only several degenerated nerve fibers sparsely distributed (Figure 6FII-III, 6GII-III).

\section{Time course of neuronal damage}

hSOD1-G93A mice showed their first sign of disease (limb tremor) at 90-100 days of age, and became moribund at 131-138 days of age, which was associated with profound degeneration of VR, DR and DF, as described before. However, histological features of degeneration in VR, DR and DF were visible as early as 60 days of age, and the degeneration deteriorated progressively thereafter (Figure 7). Thus, histological abnormalities occurred long before the animals showed any signs of disease. The neurons in DRG were not examined at the early time points, because we did not detect a significant loss of neurons at end stage.

\section{Discussion}

Transgenic mice and rats expressing mutated human SOD1 develop an ALS-like disorder and have been widely used as ALS models. Our present study shows that expression of hSOD1-G93A in mice causes damage to spinal cord sensory neurons and axons in addition to causing damage to motor neurons and axons. This is significant, because several reports have shown the impairment of the sensory nervous system in a significant percentage of ALS patients (Dyck et al., 1975; Georgesco et al., 1994; Kawata et al., 1997; Matsumoto et al., 1999; Pugdahl et al., 2007). Although degeneration of spinal cord DR axons in these animals was briefly noted in several previous reports (Gurney et al., 1994; Fischer et al., 2005), the present study
VR
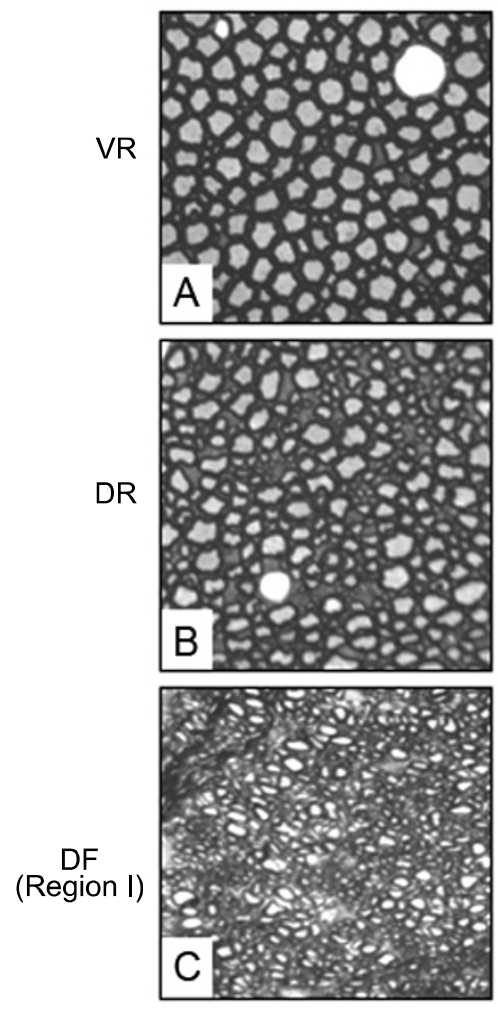

Age (days) of hSOD1-G93A mice
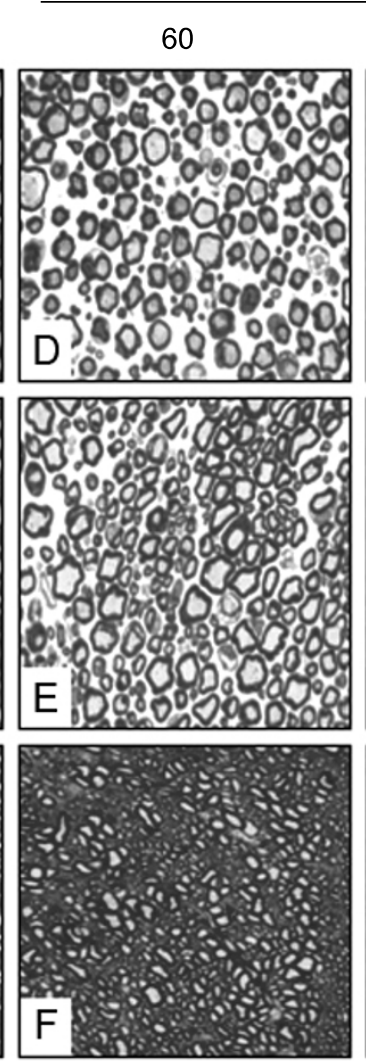

120
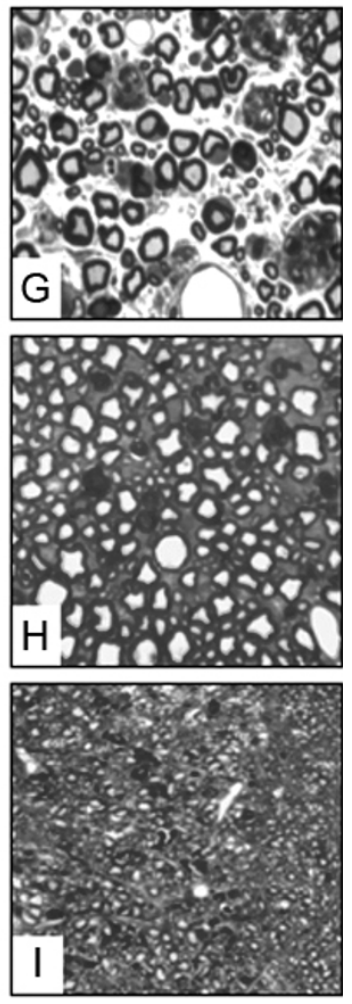

Figure 7. Time course of degeneration of VR, DR and DF in hSOD1-G93A mice. Tissues were obtained from hSOD1G93A mice at indicated ages and from their non-transgenic littermates at 120 days of age and stained with toluidine blue. The data are representative of all animals examined, at least 3 animals in each group at each time point. A-l: $400 \times$. 
provides new and detailed information about the damages in the sensory neurons and axons and compared these damages to those in motor neurons and axons, as discussed below. Moreover, our present findings appear to contradict an existing hypothesis that motor neuron degeneration in hSOD1-G93A mice begins at muscle and distal axons and proceeds in a "dying back" manner (Fischer et al., 2004; Kraft et al., 2007), because such a mechanism does not explain the parallel degeneration occurring in the sensory neurons and axons.

We focused the current study on the lumbar region of the spinal cord, since clinical presentation of hSOD1-G93A transgenic mice was primarily associated with motor neuron degeneration in this region. The animals at the end stage showed marked loss of ventral horn motor neurons and extensive damage in VR, DR, DRG and DF, among which VR damage was the most pronounced. Interestingly, the dorsal horn neurons were largely spared. The reason for the resistance of these neurons to the toxic effect of hSOD1-G93A is unknown.

Further examination of VR, DR and DF showed that tissue damage was already visible at 60 days of age, long before the animals showed any signs of disease, and VR damage was again the most evident (Figure 7).

The VR fibers originate from ventral horn motor neurons, whereas the DR fibers originate from DRG sensory neurons. Previous studies have shown that mitochondrial damage and caspase-mediated apoptosis of spinal cord motor neurons play an important role in motor neuron degeneration in hSOD1-G93A mice (Li et al., 2000; Inoue et al., 2003). We have shown some similar changes in DRG neurons, including cytoplasmic vacuolation, and mitochondrial swelling (Figure 5). In this connection, it is worth noting that a previous study showed increased number of inclusion bodies in DRG neurons of sporadic ALS patients (Sasaki et al., 2007). The VR and DR fibers exhibited Wallerian-like degeneration: axon damage, myelin degeneration, and phagocytic cell (macrophages) infiltration into the tissues (Figure 2). Tissue infiltration of macrophages was further confirmed by the presence of many CD68-positive cells (Figure 3 ). Although Wallerian degeneration often occurs as a result of axotomy, this may be a result of neuron cell death in hSOD1-G93A mice.

DF damage in hSOD1-G93A mice occurred primarily in Region I (Figure 6), which contains largediameter myelinated axons originating in DRG (Marsala et al., 2007). This finding is consistent with damage of DRG and DR in these animals. The main bulk of large myelinated axons in the DF are responsible for transmitting discriminative touch, vibratory sensibility, position sense and proprioception. Therefore, it is of note that diminished vibration sense and/or abnormal touch-pressure sensation were also detected in a significant percentage of ALS patients (Dyck et al., 1975; Georgesco et al., 1994; Kawata et al., 1997; Matsumoto et al., 1999; Pugdahl et al., 2007). The nature of the nerve fibers in Region II is unclear, but it appeared to contain small-caliber axons and did not show significant damage in hSOD1-G93A mice (Figure 4). Region III is believed to be occupied by corticospinal tract and was not significantly affected in hSOD1-G93A mice either (Figure 6), consistent with the notion that these animals present largely a disease of spinal cord motor neurons.

\section{Methods}

\section{Animals}

Transgenic hSOD1-G93A mice and their non-transgenic normal littermates were generated by breeding male hemizygous carriers (B6SJL-Tg(SOD1-G93A)1Gur/J) to female B6SJLF1 hybrids. The transgene in B6SJL-Tg (SOD1-G93A)1Gur/J mice, which were purchased from the Jackson Laboratories (Bar Harbor, ME), carried a high copy number of hSOD1-G93A. B6SJLF1 hybrids were purchased from Beijing Vital River Experimental Animal Co (Beijing, China). PCR-based genotyping of tail/ blood DNA, as previously described (Gurney et al., 1994), was used to identify the transgenic mice and their normal littermates. The animals were housed in a room with $12 \mathrm{~h}$ light cycle and provided with free access to water and diet (standard diet purchased from Beijing Vital River Experimental Animal Co. Ltd, Beijing, China).

To monitor disease progression, the animals were visually inspected daily, and weighed twice a week starting 12 weeks of age. Animals were killed at several time points to obtain spinal cord tissues for analysis. They were anesthetized with $10 \%$ chloral hydrate $(0.2 \mathrm{ml} / \mathrm{mice})$ and perfused transcardially with $4 \%$ paraformaldehyde and $0.05 \%$ glutaraldehyde in $0.1 \mathrm{M}$ phosphate buffer, $\mathrm{pH}$ 7.4. The spinal cords were carefully dissected. The lumbar enlargements (L3-5), as well as associated ventral root (VR), dorsal root (DR) and dorsal root ganglia (DRG) were fixed in $4 \%$ paraformaldehyde, or incubated in $4 \%$ glutaraldehyde for $24 \mathrm{~h}$ and then in $1 \%$ osmium tetroxide for $1 \mathrm{~h}$. All experiments were carried out in accordance with the regulation of laboratory animal management promulgated by the Ministry of Science and Technology of the People's Republic of China (1988, No. 134), which conforms to the Guidelines laid down by the NIH in the US regarding the care and use of animals for experimental procedures.

\section{Toluidine blue stain and transmission electron microscopy}

Tissues after treatment with glutaraldehyde and osmium 
tetroxide as mentioned above were dehydrated through graded acetones and embedded in epoxy resin Epon 812. Semithin sections $(1 \mu \mathrm{m})$ were cut, placed on the slides, and oven dried. The slides were stained with $1 \%$ toluidine blue solution for $10 \mathrm{~min}$, rinsed with water, dehydrated and mounted. For transmission electron microscopy examination, ultra-thin sections $(70 \mathrm{~nm})$ were cut, placed on a copper grid, stained with uranyl acetate and lead citrate, and evaluated under a JEM-1230 electron microscope.

\section{Nerve fiber teasing}

VR and DR tissues fixed in $4 \%$ paraformaldehyde were treated sequentially with $1 \%$ osmimum tetroxide for $1 \mathrm{~h}$, $33 \%$ glycerol for $12 \mathrm{~h}, 66 \%$ glycerol for $12 \mathrm{~h}$, and stored in $100 \%$ glycerol. Single nerve fibers were isolated by teasing with dissection needles under a stereo microscope (SMZ143, Motic). Images were recorded using a Nikon digital camera.

\section{Naumenko-Feigin silver stain}

VR and DR tissues fixed in $4 \%$ paraformaldehyde were paraffin-embedded and cut into $12 \mu \mathrm{m}$ sections. The sections were then deparaffinized, hydrated and impregnated in $0.14 \%$ silver nitrate at $56-60^{\circ} \mathrm{C}$ for $24 \mathrm{~h}$. After further treatment in $2 \%$ sodium sulfate and $1 \%$ hydroquinone for $6 \mathrm{~min}$, the sections were thoroughly washed in water for $15 \mathrm{~min}$ and then toned with $1 \%$ gold chloride for 5 min. After a brief rinse with water, the sections were treated with freshly prepared $2 \%$ oxalic acid for $10 \mathrm{~min}$, followed by water rinsing again. The sections were then incubated in $5 \%$ sodium thiosulfate for $3 \mathrm{~min}$ and after rinsing with water placed in $0.1 \%$ Luxol Fast Blue solution at $56-60^{\circ} \mathrm{C}$ overnight. The sections were rinsed with $95 \%$ ethanol to remove excess stain and further treated alternating between $0.5 \%$ lithium carbonate and $70 \%$ ethanol until individual fibers were distinguishable. Sections were then dehydrated and mounted.

\section{Axonal counting}

To quantify the number of axons, toluidine blue stained transverse semithin sections of lumbar ventral and dorsal roots were captured with Image-Pro Plus 5.0 software (Media Cybernetics Inc., Bethesda, MD) at $400 \times$ magnification. Three ventral and dorsal roots from three mice in each group were analysed. In normal nerve fibers, the axon appears as a sharply demarcated lightly stained area, with a few punctate regions representing mitochondria, surrounded by its homogenously dark myelin sheath. Nerve fibers with axonal degeneration or disappearance, or collapse and fragmentation of myelin were classified as degenerating fibers, and the data were analyzed statistically using Student's $t$-test.

\section{Immunohistochemistry}

Paraffin-embedded sections of VR, DR and DRG were cut at $5 \mu \mathrm{m}$, and free-floating sections of lumbar enlargement were cut at $20 \mu \mathrm{m}$. The sections were incubated in $3 \%$ $\mathrm{H}_{2} \mathrm{O}_{2}$ in methanol for $15 \mathrm{~min}$ at room temperature to block endogenous peroxidase. Non-specific antibody binding was blocked by incubating the tissues for $1 \mathrm{~h}$ at room temperature in a solution containing $10 \%$ serum obtained from the same species from which the secondary antibody was prepared. The sections were then incubated overnight at $4^{\circ} \mathrm{C}$ with a desired antibody, including monoclonal SMI-31 (Sternberger, 1:1,000 dilution), monoclonal SMI-32 (Sternberger, 1:1,500), a monoclonal antibody against CD68 (Dako, 1:25), and a polyclonal antibody against calretinin (Santa Cruz, 1:200). The sections were subsequently incubated with a biotin-conjugated secondary antibody for $1 \mathrm{~h}$ at room temperature, followed by incubation with HRPconjugated streptavidin for $1 \mathrm{~h}$ at room temperature and treatment with $0.03 \%$ diaminobenzidine as a chromogen for $10 \mathrm{~min}$.

\section{Statistical analysis}

Results are expressed as means $\pm S D$. Statistical analyses were performed using one-way ANOVA followed by Student's $t$-test. Differences were considered significant at $P<0.05$.

\section{References}

Andersen PM. Amyotrophic lateral sclerosis genetics with Mendelian inheritance. In Amyotrophic lateral sclerosis (Brown Jr RH, Swash M, Pasinelli P, eds), 2006, 187-207, Informa Healthcare, Lodon

Dyck PJ, Stevens JC, Mulder DW, Espinosa RE. Frequency of nerve fiber degeneration of peripheral motor and sensory neurons in amyotrophic lateral sclerosis. Morphometry of deep and superficial peroneal nerves. Neurology 1975; 25:781-5

Feeney SJ, McKelvie PA, Austin L, Jean-Francois MJ, Kapsa $\mathrm{R}$, Tombs SM, Byrne E. Presymptomatic motor neuron loss and reactive astrocytosis in the SOD1 mouse model of amyotrophic lateral sclerosis. Muscle Nerve 2001;24:1510-9

Fischer LR, Culver DG, Tennant P, Davis AA, Wang M, Castellano-Sanchez A, Khan J, Polak MA, Glass JD. Amyotrophic lateral sclerosis is a distal axonopathy: evidence in mice and man. Exp Neurol 2004;185:232-40

Fischer LR, Culver DG, Davis AA, Tennant P, Wang M, Coleman M, Asress S, Adalbert R, Alexander GM, Glass JD. The WIdS gene modestly prolongs survival in the SOD1G93A fALS mouse. Neurobiol Dis 2005;19:293-300

Georgesco M, Salerno A, Carlander B, Leger JJ, Camu W, Billiard M, Cadilhac J. Somatosensory evoked potentials in amyotrophic lateral sclerosis and primary lateral sclerosis. Rev Neurol (Paris) 1994;150:292-8

Gurney ME, Pu H, Chiu AY, Dal Canto MC, Polchow CY, Alexander DD, Caliendo J, Hentati A, Kwon YW, Deng HX et al. Motor neuron degeneration in mice that express a human $\mathrm{Cu}, \mathrm{Zn}$ superoxide dismutase mutation. Science 1994;264:1772-5

Inoue H, Tsukita K, Iwasato T, Suzuki Y, Tomioka M, Tateno M, Nagao M, Kawata A, Saido TC, Miura M et al. The crucial role of caspase- 9 in the disease progression of a transgenic 
ALS mouse model. Embo J 2003;22:6665-74

Isaacs JD, Dean AF, Shaw CE, Al-Chalabi A, Mills KR, Leigh $\mathrm{PN}$. Amyotrophic lateral sclerosis with sensory neuropathy: part of a multisystem disorder? J Neurol Neurosurg Psychiatry 2007;78:750-3

Jaarsma D, Haasdijk ED, Grashorn JA, Hawkins R, van Duijn W, Verspaget HW, London J, Holstege JC. Human Cu/Zn superoxide dismutase (SOD1) overexpression in mice causes mitochondrial vacuolization, axonal degeneration, and premature motoneuron death and accelerates motoneuron disease in mice expressing a familial amyotrophic lateral sclerosis mutant SOD1. Neurobiol Dis 2000;7:623-43

Kawata A, Kato S, Hayashi H, Hirai S. Prominent sensory and autonomic disturbances in familial amyotrophic lateral sclerosis with a Gly93Ser mutation in the SOD1 gene. J Neurol Sci 1997;153:82-5

Kraft AD, Resch JM, Johnson DA, Johnson JA. Activation of the Nrf2-ARE pathway in muscle and spinal cord during ALS-like pathology in mice expressing mutant SOD1. Exp Neurol 2007;207:107-17

Li M, Ona VO, Guegan C, Chen M, Jackson-Lewis V, Andrews LJ, Olszewski AJ, Stieg PE, Lee JP, Przedborski S et al. Functional role of caspase- 1 and caspase- 3 in an ALS transgenic mouse model. Science 2000;288:335-9

Marsala J, Lukacova N, Kolesar D, Sulla I, Galik J, Marsala $M$. The distribution of primary nitric oxide synthase- and parvalbumin-immunoreactive afferents in the dorsal funiculus of the lumbosacral spinal cord in a dog. Cell Mol Neurobiol 2007;27:475-504

Matsumoto A, Kawashima A, Doi S, Moriwaka F, Tashiro K. The spinal somatosensory evoked potentials in amyotrophic lateral sclerosis in relation to the spinal cord conduction velocities. No To Shinkei 1999;51:41-7

Pugdahl K, Fuglsang-Frederiksen $A$, de Carvalho $M$, Johnsen B, Fawcett PR, Labarre-Vila A, Liguori R, Nix WA,
Schofield IS. Generalised sensory system abnormalities in amyotrophic lateral sclerosis: a European multicentre study. J Neurol Neurosurg Psychiatry 2007;78:746-9

Rosen DR, Siddique T, Patterson D, Figlewicz DA, Sapp P, Hentati A, Donaldson D, Goto J, O'Regan JP, Deng HX et al. Mutations in $\mathrm{Cu} / \mathrm{Zn}$ superoxide dismutase gene are associated with familial amyotrophic lateral sclerosis. Nature 1993;362:59-62

Rabizadeh S, Gralla EB, Borchelt DR, Gwinn R, Valentine JS, Sisodia S, Wong P, Lee M, Hahn H, Bredesen DE. Mutations associated with amyotrophic lateral sclerosis convert superoxide dismutase from an antiapoptotic gene to a proapoptotic gene: studies in yeast and neural cells. Proc Natl Acad Sci USA 1995;92:3024-8

Sasaki S, Horie Y, Iwata M. Mitochondrial alterations in dorsal root ganglion cells in sporadic amyotrophic lateral sclerosis. Acta Neuropathol 2007;114:633-9

Sun W, Funakoshi H, Nakamura T. Overexpression of HGF retards disease progression and prolongs life span in a transgenic mouse model of ALS. J Neurosci 2002;22: 6537-48

Tu PH, Raju P, Robinson KA, Gurney ME, Trojanowski JQ, Lee VM. Transgenic mice carrying a human mutant superoxide dismutase transgene develop neuronal cytoskeletal pathology resembling human amyotrophic lateral sclerosis lesions. Proc Natl Acad Sci USA 1996;93:3155-60

Zang DW, Cheema SS. Degeneration of corticospinal and bulbospinal systems in the superoxide dismutase 1(G93A $\mathrm{G} 1 \mathrm{H}$ ) transgenic mouse model of familial amyotrophic lateral sclerosis. Neurosci Lett 2002;332:99-102

Zhang B, Tu P, Abtahian F, Trojanowski JQ, Lee VM. Neurofilaments and orthograde transport are reduced in ventral root axons of transgenic mice that express human SOD1 with a G93A mutation. J Cell Biol 1997;139:1307-15 